\title{
Controle da hipertensão arterial sistêmica na população negra no Maranhão: problemas $e$ desafios
}

\section{Systemic hypertension control in black population in Maranhão, Brazil: problems and challenges}

\author{
István van Deursen Varga \\ Universidade Federal do Maranhão. Centro de Ciências Humanas. \\ Departamento de Sociologia e Antropologia. São Luís, MA, Brasil. \\ E-mail: ivarga®uol.com.br

\section{Raimundo Luís Silva Cardoso} \\ Universidade Federal do Maranhão. Centro de Ciências Biológicas \\ e da Saúde. Programa de Pós-Graduação em Saúde e Ambiente. \\ São Luís, MA, Brasil. \\ E-mail: railuscaळgmail.com
}

\section{Correspondência}

István van Deursen Varga

Alameda $\mathrm{E}, \mathrm{s} / \mathrm{n}$, condomínio Brisas Altos do Calhau, edifício Brisa do Campo, ap. 1506. Altos do Calhau, São Luís, MA, Brasil. CEP 65070-628.

\section{Resumo}

Apresentamos e discutimos as circunstâncias da construção e uma parte do impacto entre profissionais e gestores de serviços de saúde e entre movimentos sociais, no Maranhão, de um texto elaborado por integrantes do Núcleo de Extensão e Pesquisa com Populações e Comunidades Rurais, Negras Quilombolas e Indígenas (NuRuNI), do Programa de Pós-Graduação em Saúde e Ambiente (PPGSA), da Universidade Federal do Maranhão (UFMA), sobre problemas identificados no controle da hipertensão arterial entre negros e sobre os desafios para a efetiva implementação e operacionalização da Política Nacional de Saúde Integral da População Negra no estado. A necessidade de nossa mobilização para elaboração deste texto (cujo objetivo foi o de alertar autoridades e operadores do Sistema Único de Saúde, bem como militantes de movimentos sociais, sobre a importância e a gravidade desses problemas, uma vez que se trata da nosologia de maior prevalência no estado e no país, e das que acarretam maior número de sequelas e nosologias secundárias graves) e para intervenção no contexto das políticas de saúde no estado nos foi evidenciada em decorrência de aulas ministradas, sobre o assunto, durante o Curso de Especialização em Saúde da Mulher Negra, promovido pelo NuRuNI/PPGSA/ UFMA, com apoio da Coordenação-Geral de Saúde das Mulheres, do Departamento de Ações Programáticas Estratégicas, da Secretaria de Atenção à Saúde, do Ministério da Saúde, com financiamento do Fundo Nacional de Saúde.

Palavras-chave: Controle da Hipertensão Arterial na População Negra; Saúde da População Negra; Saúde da Mulher Negra. 


\section{Abstract}

We present and discuss the circumstances of the construction and a part of the impact among professionals and managers of health services and social movements, in Maranhão, Brazil, of a text drawn up by members of the Núcleo de Extensão e Pesquisa com Populações e Comunidades Rurais, Negras Quilombolas e Indígenas [Research and Extension Center of Rural, Black Quilombolas, and Indigenous Populations and Communities] (NuRuNI), of the Programa de Pós-Gradução em Saúde e Ambiente [Graduate Program in Health and Environment] of the Federal University of Maranhão (UFMA), on problems identified in the control of hypertension among blacks, and the challenges for the effective implementation and operationalization of the National Policy of Integral Health of the Black Population in the state. The need for our mobilization for the elaboration of this text (which objective was to alert the authorities and operators of the Brazilian Unified Health System, as well as activists from social movements, on the importance and the seriousness of these problems, because it is the nosology of higher prevalence in the state and in the country, and that entail greater number of sequels and secondary serious nosologies) and for our intervention in the context of health policies in the state has been highlighted as a result of classes on the subject, during the Graduate Specialization in Black Women's Health, promoted by NuRuNi, with the support of the Coordenação-Geral de Saúde das Mulheres [General Coordination of Women's Health] of the Ministry of Health, with funding from the Fundo Nacional de Saúde [National Health Fund].

Keywords: Control of Hypertension in the Black Population; Health of the Black Population; Black Women's Health.

\section{Introdução}

0 relato que segue pretende servir de alerta e suscitar reflexões entre os que, de algum modo, pesquisam ou atuam profissionalmente nos campos da Atenção Básica de Saúde, da Estratégia de Saúde da Família (ESF), da Atenção Farmacêutica, da Saúde da População Negra, da Saúde da Mulher, ou, mais especificamente, no Sistema de Gestão Clínica de Hipertensão Arterial e Diabetes Mellitus da Atenção Básica (Hiperdia).

Apresentamos, inicialmente, o texto divulgado entre profissionais e gestores de serviços de saúde e entre movimentos sociais, em São Luís (MA), elaborado como parte dos encaminhamentos deliberados na reunião de 26 de agosto de 2010 , realizada no auditório do Programa de Pós-Graduação em Saúde e Ambiente (PPGSA) da Universidade Federal do Maranhão (UFMA), entre integrantes do Núcleo de Extensão e Pesquisa com Populações e Comunidades Rurais, Negras Quilombolas e Indígenas (NuRuNI) do PPGSA/UFMA, representantes de entidades do movimento negro e de comunidades tradicionais de terreiros de São Luís (MA). Esse texto foi elaborado a partir das contribuições de István van Deursen Varga (NuRuNI/UFMA), Maria dos Remédios Lira (Secretaria Municipal de Saúde de São Luís), Raimundo Luís Silva Cardoso (NuRuNI/UFMA), Zelena Maria Pereira dos Santos (NuRuNI/UFMA - in memoriam), Antonio Henrique França Costa (NuRuNI/ UFMA) e Rôlzele Robson Marques (NuRuNI/UFMA), presentes à referida reunião, tendo sido finalizado em 14 de dezembro de 2010.

Prosseguimos com a apresentação de algumas das discussões suscitadas e de suas repercussões, e concluímos com algumas recomendações (e indagações), especialmente aos gestores dessas políticas públicas.

\section{Saúde da população negra no Brasil e no Maranhão}

No Brasil, embora documentos oficiais venham reconhecendo e tomando a saúde da população negra como objeto de políticas públicas específicas (como de saúde), esse reconhecimento tem encontrado resistências tanto para se firmar junto dos aparelhos formadores (universidades e escolas técnicas 
de saúde, sobretudo) quanto para se materializar operacionalmente no âmbito do Sistema Único de Saúde (SUS), das políticas e serviços do Ministério e das secretarias estaduais e municipais de saúde.

Essa especificidade do campo da saúde da população negra deve-se, de imediato, a algumas nosologias e condicionantes sociais, que acometem de modo bastante diferenciado a população negra:

- especificidades genéticas - caso da anemia falciforme e da deficiência da glicose6fosfato desidrogenase que, no Brasil, afetam, predominantemente, essa população;

- especificidades clínicas - caso da hipertensão arterial sistêmica e do diabetes mellitus, cujos quadros mais graves e de maior resistência aos protocolos terapêuticos atualmente em uso são bem mais frequentes nessa população;

- especificidades sociais - em decorrência da histórica marginalização, das piores condições de vida e do racismo a que a população negra vem sendo submetida no país há mais de quatro séculos, algumas das ditas endemias negligenciadas, como a tuberculose, a violência policial, as dificuldades de acesso e o mau atendimento nos serviços públicos em geral, e mais especificamente nos de saúde, também a têm majoritariamente vitimado.

Os primeiros movimentos e articulações do Governo Federal com vistas à definição de uma política específica de atenção à saúde da população negra tiveram início apenas em 1995, em resposta às demandas da Marcha Zumbi dos Palmares Contra o Racismo, pela Cidadania e pela Vida (Programa das Nações Unidas para o Desenvolvimento e Organização Pan-Americana da Saúde 2002). A Política Nacional de Saúde Integral da População Negra foi, finalmente, formalizada pela Portaria ${ }^{0} 992$ do Ministro da Saúde, de 13 de maio de 2009 (publicada no Diário Oficial da União nº 9o, de 14 de maio de 2009).

\section{Controle da hipertensão arterial na população negra}

No que se refere especificamente à política de controle da hipertensão arterial entre a população negra, além da falta de capacitação específica de recursos humanos, enfrentam-se o problema de protocolos terapêuticos inadequados e a frequente descontinuidade da distribuição dos medicamentos disponibilizados pela rede de serviços do SUS.

A literatura vem demonstrando que justamente duas das drogas mais prescritas para seu controle, e que fazem parte da lista de medicamentos básicos do Ministério da Saúde - o Captopril e o Maleato de Enalapril, do grupo dos anti-hipertensivos inibidores da enzima conversora da angiotensina (iECA) - estão contraindicadas para pessoas negras hipertensas. Segundo o próprio Ministério da Saúde

Nos negros, a prevalência e a gravidade da hipertensão são maiores, o que pode estar relacionado a fatores étnicos e/ou socioeconômicos. Em nosso país predominam os miscigenados, que podem diferir dos negros quanto às características da hipertensão. Não há evidências de ação diferenciada das drogas anti-hipertensiva em nossa população. Entretanto, estudos recentes em populações de indivíduos negros norte-americanos, o uso de iECA se mostraram menos eficazes, especialmente na prevenção de AVC, que outras classes de anti-hipertensivos. Devendo, portanto, não serem considerados de primeira escolha nesta população (BRASIL, 2006, p. 33).

\section{Controle da hipertensão arterial na população negra no Maranhão: para um estudo de caso}

A preocupação com a inadequação, para a população negra, do protocolo terapêutico em uso para controle da hipertensão arterial na rede de serviços do SUS tomou corpo, no âmbito do NuRuNI, em setembro de 2009, após aulas sobre esse assunto ministradas pela doutora Fátima Oliveira, no curso de especialização em saúde da mulher negra promovido pelo PPGSA/UFMA - projeto apoiado pela Coordenação-Geral de Saúde das Mulheres, do Ministério da Saúde, com financiamento do Fundo Nacional de Saúde.

À época, tendo em vista o trabalho que vinha sendo desenvolvido com a comunidade quilombola de Jamary dos Pretos, em Turiaçu (MA), a coordenação 
do NuRuNI fez contato com o Secretário Municipal de Saúde de Turiaçu, propondo uma capacitação específica, para controle da hipertensão arterial da população negra, das equipes da ESF que estavam sendo contratadas pelo município. Contatos com vários especialistas nesse campo foram então realizados pelo NuRuNI, com o objetivo de amadurecer o projeto desse curso de capacitação. À medida que esses contatos e mais estudos eram realizados no âmbito do NuRuNI, tornava-se mais claro que o problema e os desafios a enfrentar eram maiores do que inicialmente pareciam, uma vez que a compra dos itens da lista de medicamentos básicos disponibilizados pela rede de serviços do SUS era centralizada no Ministério da Saúde: tratava-se de problema de âmbito e repercussões federais, estaduais e municipais, cuja superação exigiria respostas nesses três níveis de gestão do SUS.

Após alguns contatos informais com técnicos da Secretaria de Estado da Saúde do Maranhão, que não surtiram efeitos, em 17 de agosto de 2010 o coordenador do NuRuNI encaminhou ao Secretário de Estado da Saúde, à Secretária de Estado da Igualdade Racial e ao presidente do Conselho Estadual da Igualdade Racial (Ceir) do Maranhão os Ofícios NuRuNI n ${ }^{0}$ o62, o63 e o64.

Os ofícios alertavam essas autoridades para o fato de que, num estado em que a população negra é majoritária, a Política Nacional de Saúde Integral da População Negra não vinha sendo implementada adequadamente, em vários aspectos:

- no que se referia à capacitação do quadro de profissionais do SUS, tanto das equipes do ESF como de todos os níveis de atenção à saúde;

- no que se referia à população quilombola, a situação era ainda mais grave, dada sua vulnerabilidade institucional, que se expressa nas dificuldades de acesso a serviços e na frequente deficiência ou mesmo ausência de políticas municipais específicas a elas voltadas (de promoção da saúde, acesso à água potável, saneamento básico, educação, proteção e recuperação ambiental etc.), além das grandes dificuldades enfrentadas na efetiva titulação de seus territórios;
- no que se referia especificamente à política de controle da hipertensão arterial entre a população negra, além da falta de capacitação específica de recursos humanos enfrentavase o problema de protocolos terapêuticos inadequados (citava-se que a literatura vinha demonstrando que o Captopril e Maleato de Enalapril, duas das drogas mais prescritas para esse controle e que fazem parte da lista de medicamentos básicos do Ministério da Saúde, estão contraindicadas para pessoas negras hipertensas), além da frequente descontinuidade da distribuição desses medicamentos.

Os ofícios se concluíam com a proposta de realização de uma primeira reunião com essas autoridades e equipes, com vistas à definição de possí veis frentes de colaboração, nesse âmbito, entre a Secretaria de Estado da Saúde do Maranhão e o NuRuNI.

Enquanto aguardava resposta aos ofícios, a coordenação do NuRuNI entrou em contato com entidades do movimento negro e das comunidades tradicionais de terreiros de São Luís, convidando-as para uma reunião sobre o assunto, a ser realizar em 26 de agosto de 2010, no auditório do PPGSA/ UFMA. Participaram dessa reunião, além dos integrantes do NuRuNI, representantes do Conselho Municipal das Populações Afro-Descendentes de São Luís (Comafro), do Ceir, da Secretaria de Estado da Saúde do Maranhão/Programa de DST/aids; do Núcleo de Estudos e Pesquisas Ambientais (Nepa) da UFMA; do Movimento Negro Unificado (MNU); do Fórum Permanente de Educação e Diversidade Étnico-Racial do Maranhão; da Rede de Religiões Afro-Brasileiras e Saúde - Núcleo Maranhão; da Organização Consciência Negra; do Centro de Cultura Negra do Maranhão (CCN-MA); do Centro de Formação para a Cidadania Akoni; da Associação das Comunidades Negras Rurais e Quilombolas do Estado do Maranhão (Aconeruq); da Associação de Saúde da Periferia do Maranhão (ASP-MA); de alunas do curso de especialização em saúde da mulher negra da UFMA; de alunos do mestrado em Saúde e Ambiente da UFMA.

Seguem os relatos, apresentados na referida reunião de 26 de agosto de 2010 , da experiência 
de integrantes de duas diferentes equipes da ESF em São Luís:

Na equipe 66 da Estratégia Saúde da Família tem cerca de 140 hipertensos cadastrados e acompanhados. Grande parte desses pacientes é negra el ou miscigenada, comumente faz uso de Captopril e/ou Enapril, e ocorreu que por meses (desde maio até aproximadamente julho de 2010) esses medicamentos ficaram escassos, chegando até ao desaparecimento, principalmente o Captopril (iECA), concomitantemente à disponibilização de outros anti-hipertensivos, como o Atenolol (do grupo dos bloqueadores beta-adrenérgicos) e o Pressat (do grupo dos inibidores do fluxo de cálcio), entre outros, fato que levou o médico da equipe a mudar a terapêutica medicamentosa (iniciou a prescrever os medicamentos que estavam disponíveis). O que se observou, na prática, foi uma melhora no controle pressórico de muitos pacientes e também uma diminuição na ingestão de comprimidos: alguns pacientes que faziam uso de até seis comprimidos de Captopril por dia, após a mudança medicamentosa, passaram a tomar só um comprimido de Pressat por dia.

Nossa inquietação: agora voltou a estar disponivel o Captopril e não sabemos se teremos os outros anti-hipertensivos para manter o tratamento, pois a população que atendemos se encontra em condições economicamente desfavoráveis e às vezes não possui dinheiro para manter o uso das medicação sem que sejam fornecidas pelo sistema (Informação verbal).

Este relato busca socializar a experiência quanto à mudança de esquema terapêutico dos hipertensos negros que são atendidos por uma equipe da Estratégia Saúde da Família (ESF) do município de São Luis.

Há um ano, quando assumi a função de enfermeiro da ESF, observava que a maioria dos hipertensos negros que eram atendidos na unidade de saúde, que utilizavam como esquema terapêtico os inibidores da enzima conversora de angiotensina (ECA) - comumente o Captopril - não conseguiam desenvolver estado de normalidade pressórico, mesmo relatando que utilizavam corretamente os medicamentos.

A partir dessa realidade, agendei sucessivos encontros com a médica da equipe para discussão da efetividade dos inibidores da ECA na população negra, à luz do que preconiza o Caderno de Atenção Básica do Ministério da Saúde 15 que contraindica esses fármacos para o segmento populacional negro, devido à sua pouca eficiência. Dessa forma, paulatinamente, ela foi mudando o esquema terapêutico desses usuários com intuito de melhorar a resposta terapêutica no tratamento da hipertensão. Transcorridos aproximadamente seis meses, obtivemos uma melhora e manutenção de normalidade dos níveis pressóricos de 80\% da população negra assistida (Informação verbal).

\section{Controle da hipertensão arterial na população negra: repercussões em São Paulo}

Esse processo, desencadeado no Maranhão, ainda em 2010 repercutiu significativamente também em São Paulo: o professor doutor Luís Eduardo Batista - docente do Curso de Especialização em Saúde da Mulher Negra do PPGSA/UFMA e então coordenador da área técnica Saúde da População Negra, do Grupo Técnico de Ações Estratégicas (GTAE), da Coordenadoria de Planejamento em Saúde, da Secretaria de Estado da Saúde de São Paulo - em 12 de dezembro de 2010 divulgava nota técnica dirigida à doutora Maria Cristina Megid, do Centro de Vigilância Sanitária (CVS), da Coordenadoria de Controle de Doenças, da Secretaria de Estado da Saúde de São Paulo, cujo texto principal anexamos ao fim deste artigo.

\section{Desafios e propostas}

Entendemos que esses relatos e informações indicam, claramente, obstáculos a superar, e temos algumas propostas na direção da efetiva implementação, no Maranhão e em São Paulo, da Política Nacional de Saúde Integral da População Negra e 
de uma política de atenção farmacêutica à saúde voltada à diversidade de necessidades da população:

- maior investimento das universidades e centros de pesquisa em pesquisas clínicas, com recorte racial/ étnico, sobre a eficácia e a adequação dos anti-hipertensivos, bem como dos demais medicamentos da farmácia básica;

- que o Ministério da Saúde promova, imediatamente, uma revisão da lista dos antihipertensivos da farmácia básica por ele adquiridos, tendo em vista as necessidades específicas da população negra;

- que o Ministério da Saúde passe a realizar, a curtíssimo prazo, oficinas de construção e atualização de um consenso terapêutico sobre controle da hipertensão arterial na população negra;

- que o Ministério da Saúde promova oficinas de capacitação e atualização em saúde da população negra para todas as equipes da ESF no país;

- que secretarias estaduais e municipais de saúde cumpram o determinado pela Portaria de descentralização dos recursos da farmácia básica, adquirindo diretamente medicamentos demandados pelas necessidades específicas de seus usuários.

\section{Referências}

BATISTA, L. E. Nota técnica à dra. Maria Cristina Megid, do Centro de Vigilância Sanitária (CVS), da Coordenadoria de Controle de Doenças, da Secretaria de Estado da Saúde de São Paulo. São Paulo: Secretaria de Estado da Saúde de São Paulo, 2010.

BRASIL. Ministério da Saúde. Secretaria de Atenção à Saúde. Departamento de Atenção Básica. Cadernos de Atenção Básica 15: hipertensão arterial sistêmica para o Sistema Único de Saúde. Brasília, DF, 2006.

POLI-DE-FIGUEIREDO, C. E. Hipertensão em situações especiais. Jornal Brasileiro de Nefrologia, São Paulo, v. 32, 2010. Suplemento 1.

\section{Contribuição dos autores}

Os autores contribuíram igualmente em todas as etapas de elaboração do artigo.

Recebido: $11 / 04 / 2014$

Aprovado: 03/07/2014 


\section{Anexo}

\section{Nota Técnica à dr. Maria Cristina Megid, do Centro de Vigilância Sanitária (CVS), da Coordenadoria de Controle de Doenças, da Secretaria de Estado da Saúde de São Paulo (Luís Eduardo Batista, 20ıo)}

Em evento realizado na cidade de Francisco Morato-SP, fui indagado sobre o tema hipertensão arterial sistêmica - integrante da platéia enfatizou que a população negra tem quadros mais graves de hipertensão e de maior resistência aos protocolos terapêuticos atualmente em uso - a partir destas afirmativas fez as seguintes questões (1) a Secretaria de Estado da Saúde de São Paulo tem protocolos terapêuticos que levam em consideração as especificidades desta população; (2) a SES SP tem conhecimento que o Captopril e o Maleato de Enalapril, do grupo dos anti-hipertensivos inibidores da enzima conversora da angiotensina (iECA) - são drogas que não respondem bem ao tratamento do hipertenso negro; (3) se a SES SP conhece, porque estas drogas são as únicas que estão na lista de medicamentos da atenção básica.

Frente a meu desconhecimento realizei buscas com profissionais da SES; documentos técnicos e consulta a pesquisadores.

Técnicos da CCTIES/SES SP, afirmaram que os protocolos terapêuticos em uso não dizem nada sobre eficácia de medicamentos e/ou necessidade de adequação dos protocolos da hipertensão arterial para a população negra.

O Cadernos de Atenção Básica 15 do Ministério da Saúde, Secretaria de Atenção à Saúde, Departamento de Atenção Básica. Brasília: Ministério da Saúde, 2006. p. 33 - 'X. Hipertensão em populações especiais, diz:

'Nos negros, a prevalência e a gravidade da hipertensão são maiores, o que pode estar relacionado a fatores étnicos e/ou socioeconômicos. Em nosso país predominam os miscigenados, que podem diferir dos negros quanto às características da hipertensão. Não há evidências de ação diferenciada das drogas anti-hipertensiva em nossa população. Entretanto, estudos recentes em populações de indivíduos negros norte-americanos, o uso de iECA se mostraram menos eficazes, especialmente na prevenção de AVC, que outras classes de anti-hipertensivos. Devendo portanto, não serem considerados de primeira escolha nesta população.'

A Sociedade Brasileira de Hipertensão, reiterando o acima disposto pelo Ministério da Saúde, em 20o6, em artigo publicado recentemente-2010, indica alternativas medicamentosas aos anti-hipertensivos dos grupos dos iECA:

'Os afrodescendentes em geral respondem menos à monoterapia com betabloqueadores, inibidores da enzima conversora da angiotensina (iECAs) e bloqueadores dos receptores da angiotensina (BRA) do que aos diuréticos e antagonistas dos canais de cálcio (ACC) di-hidropiridínicos. A escolha do anti-hipertensivo para tratar esse grupo étnico é norteada pela presença de comorbidades e pela eficácia em atingir as metas pretendidas. $\mathrm{O}$ uso de terapia não medicamentosa tem apresentado melhores resultados em afrodescendentes do que em brancos. Em indivíduos com proteinúria, o uso de iECA está indicado, da mesma forma como ocorre em indivíduos de cor branca.' (Sociedade Brasileira de Hipertensão. Revista Brasileira de Hipertensão, vol. 17(1): 52-58, 2010, p. 52).

No texto acima, a Sociedade Brasileira de Hipertensão ressalta um importante aspecto da questão, ao afirmar que '...o uso de terapia não medicamentosa tem apresentado melhores resultados em negros do que em brancos'. Esta constatação parece importante para os programas de controle da hipertensão arterial entre a população negra, uma vez que indica medidas não-medicamentosas como necessárias e de grande importância no controle da hipertensão arterial entre negros/as. 
Depois de realizar levantamento técnico, realizamos contato com pesquisadores para investigar se tinham algum conhecimento sobre o tema - dentre os respondentes está o prof. dr. István van Deursen Varga da Universidade Federal do Maranhão.

Prof. dr. István Varga encaminhou o relato de caso (em anexo) de uma equipe de saúde da família de um bairro periférico de São Luís do Maranhão, onde constatou-se que:

- a equipe 66 da ESF de São Luís do Maranhão, possui aproximadamente 14o hipertensos, estes fazem uso normalmente de Captopril e/ou Inalapril (iECA);

- recentemente estes medicamentos faltaram e os médicos utilizaram o que estava disponível o Atenolol (do grupo dos bloqueadores beta-adrenérgicos) e o Pressat (do grupo dos inibidores do fluxo de cálcio);

- o que se observou, na prática, foi uma melhora no controle pressórico de muitos pacientes e também uma diminuição na ingestão de comprimidos: alguns pacientes que faziam uso de até 6 comprimidos de Captopril por dia, após a mudança medicamentosa, passaram a tomar um comprimido de Pressat por dia;.

A preocupação agora é que a rede voltou a disponibilizar o Captopril, e não sabemos se teremos os outros anti-hipertensivos para manter o tratamento, pois a população que atendemos se encontra em condições economicamente mais desfavoráveis e às vezes não possui dinheiro para manter o uso das medicação sem que seja fornecido pelo sistema.

Considerando que: (1) temos no Estado de São Paulo uma população negra (preta mais parda) de aproximadamente 10.083.985 - 27\% da população do estado; (2) a redução das complicações e mortes relacionadas à hipertensão arterial é uma das metas inseridas no Plano Estadual de Saúde (Eixo V; objetivo 9); (3) a necessidade de aperfeiçoar o atendimento integral da hipertensão arterial, solicitamos ao Núcleo de Farmacovigilância do CVS uma investigação junto ao produtor dos medicamentos de referência relatados sobre eficácia e eventos adversos relacionados a população negra. Vossa Senhoria bem sabe a importância desta discussão para a saúde dos paulistas. Desde já agradecemos o empenho de Vossa Senhoria. 